\title{
The role of spontaneous portal-systemic shunts in liver transplantation: case report and literature review
}

\author{
Tielong Wang ${ }^{1,2,3 \#} \wedge$, Jinlong Gong ${ }^{1,2,3}$, Zhitao Chen ${ }^{1,2,3}$, Changjun Huang ${ }^{1,2,3}$, Jia Yu $^{1,2,3}$, Zhiyong Guo ${ }^{1,2,3}$, \\ Xiaoshun $\mathrm{He}^{1,2,3}$ \\ ${ }^{1}$ Organ Transplant Center, The First Affiliated Hospital, Sun Yat-sen University, Guangzhou, China; ${ }^{2}$ Guangdong Provincial Key Laboratory \\ of Organ Donation and Transplant Immunology, Guangzhou, China; ${ }^{3}$ Guangdong Provincial International Cooperation Base of Science and \\ Technology (Organ Transplantation), Guangzhou, China \\ "These authors contributed equally to this work. \\ Correspondence to: Xiaoshun He. Organ Transplant Center, The First Affiliated Hospital, Sun Yat-sen University, No. 58 Zhongshan Er Road, \\ Guangzhou 510080, China. Email: gdtrc@163.com.
}

\begin{abstract}
We described two liver transplants for patients with end-stage liver disease and spontaneous portal-systemic shunt (SPSS). We ligated the splenorenal shunt (SRS) in the first case but did not ligate it in the second case. Postoperative examination revealed significant differences in portal blood flow velocity, serum ammonia level, liver function and prognosis between two cases. The portal blood flow in the first case was sufficient with decreased serum ammonia and immediate liver graft function. The portal blood flow was insufficient and serum ammonia level was not significantly reduced after operation in the second case probably because SRS was still present after surgery. The first case recovered well after operation and was discharged uneventfully, however, the second patient suffered early allograft dysfunction (EAD) after operation and died of pulmonary infection on postoperative day (POD) 18. Proper management of SPSS in liver transplantation (LT) is important because it can affect the function of liver graft and patient prognosis, so we reviewed the relevant literature and list different types of SPSS and their clinical characteristics. We recommend that SPSS greater than $8 \mathrm{~mm}$ in diameter should be ligated in LT with non-small size graft to ensure adequate portal flow and preserved with small size liver graft to avoid portal hypertransfusion and portal hypertension except obviously insufficient portal blood flow.
\end{abstract}

Keywords: Hepatic encephalopathy (HE); liver transplantation (LT); small-for-size syndrome (SFSS); splenorenal shunt (SRS); spontaneous portal-systemic shunt (SPSS)

Submitted Dec 15, 2020. Accepted for publication Feb 05, 2021.

doi: 10.21037/apm-20-2431

View this article at: http://dx.doi.org/10.21037/apm-20-2431

\section{Introduction}

Spontaneous portal-systemic shunt (SPSS) is defined as communications spontaneously formed between the portal and systemic venous circulation. SPSS are usually formed secondary to portal hypertension caused by liver cirrhosis. SPSS may cause hepatic encephalopathy (HE) and further deterioration of liver function. In patients with end-stage liver disease who are candidates for liver transplantation (LT), the incidence of SPSS varied from $3.8 \%$ to $60 \%$ (1). SPSS might have a portal flow "stealing" effect, which could decrease the portal inflow into the graft and compromise graft function recovery (2). However, SPSS might prevent small-for-size syndrome (SFSS) by limiting the portal hypertransfusion, transient portal hypertension

^ ORCID: 0000-0003-3914-6412. 

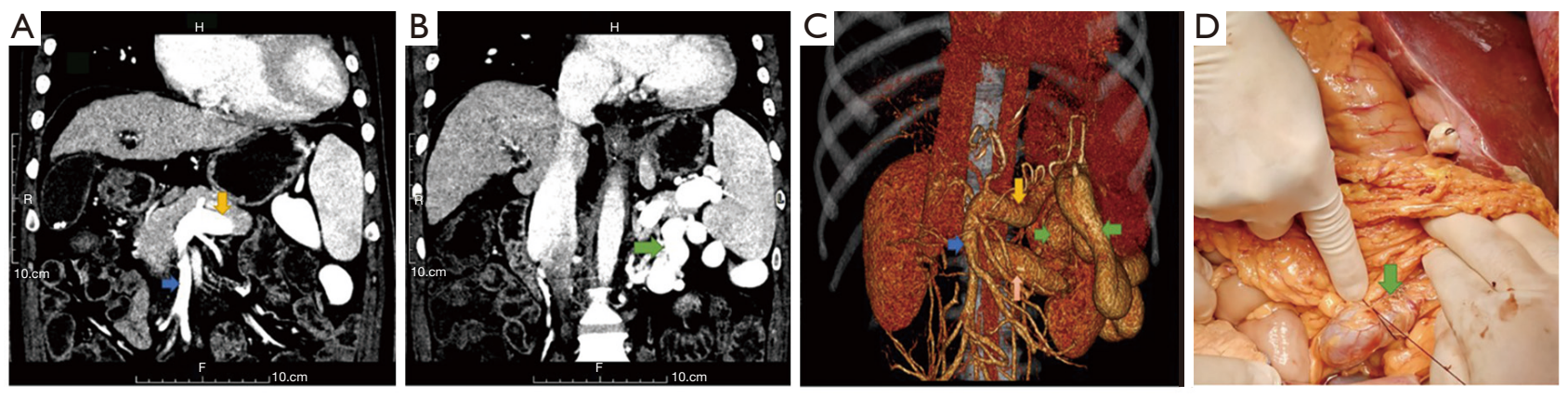

$\Rightarrow$ SWV $\Rightarrow S V$

$\Rightarrow$ SRS $\Rightarrow$ LRV

Figure 1 The perioperative image of case 1. (A) SMV and SV were well filled with contrast medium and PV was completely occlusive; (B) SRS with $1.8 \mathrm{~cm}$ in diameter between SV and LRV; (C) 3-D reconstruction of PV system showed SMV, SV, LRV and SRS between SV and LRV; (D) ligation of SRS after LT. SMV, superior mesenteric vein; SV, splenic vein; PV, portal vein; SRS, splenorenal shunt; LRV, left renal vein; LT, liver transplantation.

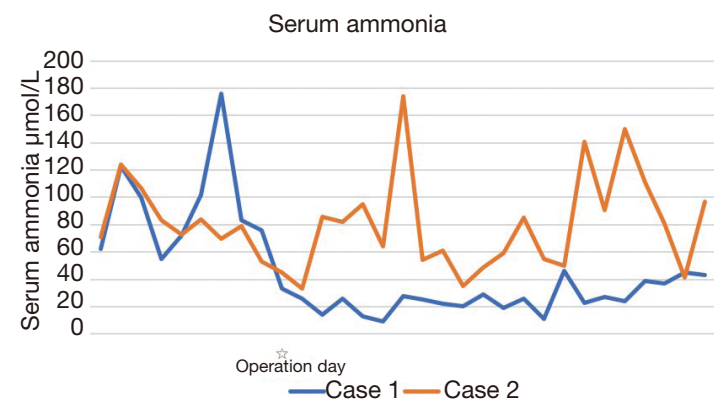

Figure 2 Postoperative serum ammonia level was lower in case 1 than that in case 2 .

and sinusoidal damage that frequently occur after revascularization of small-for-size grafts $(3,4)$. Therefore, the management of SPSS in LT is still complicated and needs to be further investigated.

We present the following article in accordance with the CARE reporting checklist (available at http://dx.doi. org/10.21037/apm-20-2431).

\section{Case presentation}

All procedures performed in studies involving human participants were in accordance with the ethical standards of the institutional and/or national research committee(s) and with the Helsinki Declaration (as revised in 2013). Written informed consent was obtained from the patient.

\section{Case 1}

A 48-year-old male patient was admitted to our center because of decompensated cirrhosis a HE (grade III) secondary to hepatitis B virus infection. He had a history of type 2 diabetes and hepatitis B for 2 years without regular treatments. The Model for End-Stage Liver Disease (MELD) score for him was 18 . The serum ammonia increased significantly (maximum $176 \mu \mathrm{mol} / \mathrm{L}$ ). CT scan revealed complete occlusion of the portal vein (PV), splenomegaly and a large splenorenal shunt (SRS) with $1.8 \mathrm{~cm}$ in diameter (Figure $1 A, B, C$ ). He received a standard orthotopic LT. Before the operation, the anesthetist found his central venous pressure (CVP) was remarkably high, reaching $30 \mathrm{cmH}_{2} \mathrm{O}$. During the operation, the SRS was found and ligated (Figure 1D). End-to-end anastomosis of $\mathrm{PV}$ was conducted after removing the old thrombus. Consequently, an efficient portal flow was obtained visually. The operation was successful, with CVP and blood ammonia decreased to normal range on postoperative day (POD) 1 after operation (Figure 2). Regular ultrasound examination found PV blood flow was adequate (Figure 3). His liver function was almost completely normal since POD 5 and he was discharged uneventfully (Figure 4).

\section{Case 2}

A 66-year-old male patient was admitted to our center 


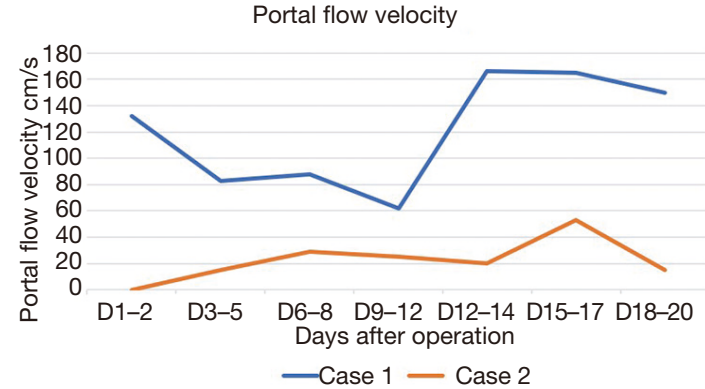

Figure 3 Postoperative PV flow velocity was higher in case 1 than that in case 2 . PV, portal vein.

because of hepatocellular carcinoma (HCC) and HE (grade III). He was diagnosed of HCC and received transcatheter arterial chemoembolization (TACE) 2 years ago. We also found portal vein thrombus (PVT) (from the junction of splenic vein $(\mathrm{SV})$ and superior mesenteric vein (SMV) to the bifurcation of the left and right branches of the PV) and a large SRS with $2 \mathrm{~cm}$ in diameter (Figure 5A). Standard orthotopic LT was performed because of progressively aggravated HE. The PV flow was satisfactory by visual examination after a $1.5 \mathrm{~cm}$ old PVT removed (Figure 5B). The SRS was not ligated, because the portal flow was sufficient before PV anastomosis. PV was then anastomosed in an end-to-end fashion without stenosis. However, on POD 2, ultrasound examination revealed no portal blood flow signal with an increased hepatic artery flow velocity (maximum velocity $210 \mathrm{~cm} / \mathrm{s}$ ). The hepatic veins and inferior vena cava (IVC) were patent. Therefore, an emergency enhanced CT scan was performed. The scan showed the PV and its branches were well filled with contrast medium without dilation or stenosis (Figure 5C). The SRS was still present, and its diameter did not decrease (Figure 5D). Regular abdominal ultrasound examination after operation showed the PV flow velocity was low (from 15 to $53 \mathrm{~cm} / \mathrm{s}$, the average velocity was $23 \mathrm{~cm} / \mathrm{s}$, Figure 3). His transaminase level peaked on POD 8 and then dropped back to normal, but his bilirubin level continued to rise, peaking at $390 \mu \mathrm{mol} / \mathrm{L}$ on POD 13. Early allograft dysfunction (EAD) was diagnosed based on bilirubin level $(229 \mu \mathrm{mol} / \mathrm{L})$ and INR value (1.63) on POD 7 . The serum ammonia level remained high after operation, possibly due to liver dysfunction and SRS (Figure 2). The patient eventually died of graft failure and a severe lung infection on POD 18 (Figure 4).

\section{Discussion}

The increasing resistance to portal outflow and a growth in splanchnic blood flow can cause portal hypertension $(5,6)$. In order to compensate the portal hypertension, splanchnic vessels frequently develop a vast variety of SPSS (7). According to their localization to left or right side of the spleno-porto-mesenteric confluence, SPSS can be classified into left-sided and right-sided (central) shunts. Left-sided shunts include gastro-renal shunt, gastro-caval shunt and SRS. Right-sided (central) shunts include meso-renal shunt, paraumbilical shunt, rectal varices and esophageal varices (8). SPSS in different types have different clinical characteristics. Gastro-renal shunt and gastro-caval shunt are characterized by gastric varices bleeding. SRS, mesorenal shunt and paraumbilical shunt are characterized by HE. Rectal varices and esophageal varices are characterized by lower gastro-intestinal bleeding or hematemesis and melena. PVT can be seen in different types of SPSS. The above symptoms may occur alone or in combination with each other.

SPSS can only alleviate the chronic portal hypertension, but it causes more damages to liver function and nervous system (7-9). Deterioration of liver function is caused by reduced portal flow or PVT. With the progression of cirrhosis and portal hypertension, the flow of portalsystemic shunt is increasing, the splanchnic vessel (splenic and mesenteric) natural outflow to PV can be taken over by exceptionally large portal-systemic shunts (with or without varices). The liver disease, portal hypertension and portalsystemic shunt become an enclosed vicious cycle (10). As the size of SPSS increases, the PV flow will decrease and become hepatofugal ultimately. PVT eventually block PV completely. Liver atrophy, liver synthetic dysfunction and $\mathrm{HE}$ would get more serious at advanced stage (11).

HE frequently occurs in patients with cirrhosis caused by accumulation of toxins due to hepatocellular dysfunction or by portal-systemic shunt $(9,12,13)$. According to the underlying disease, $\mathrm{HE}$ is subdivided into three types, including type A resulting from acute liver failure (ALF), type $\mathrm{B}$ resulting predominantly from portosystemic bypass or shunt and type $\mathrm{C}$ resulting from cirrhosis (13). Type-B HE can also occur after LT (14), that's because blood ammonia from the gastrointestinal tract enters the systemic circulation directly through the large SPSS not ligated during operation. SPSS also occurs in non-cirrhotic 


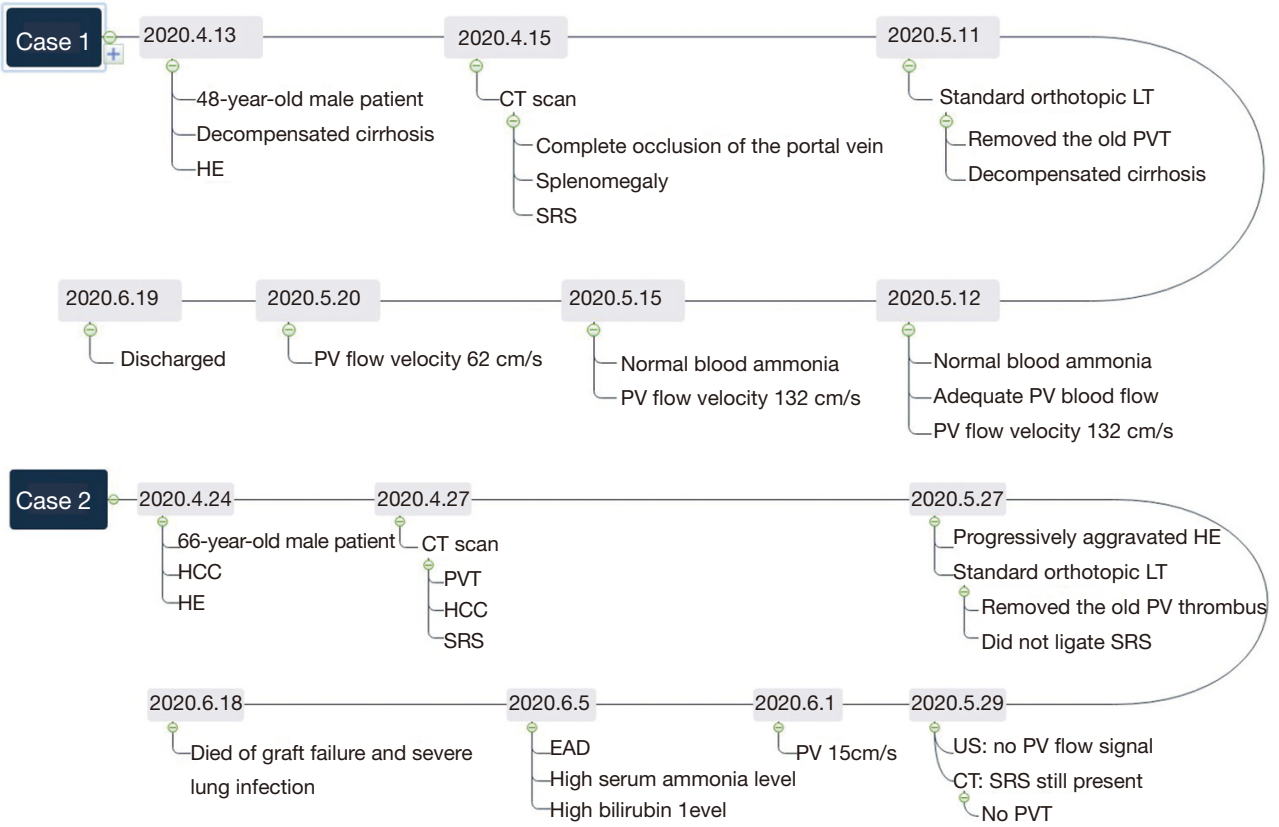

Figure 4 Timeline of the patient's therapy. HE, hepatic encephalopathy; SRS, splenorenal shunt; LT, liver transplantation; PVT, portal vein thrombus; PV, portal vein; HCC, hepatocellular carcinoma; EAD, early allograft dysfunction.
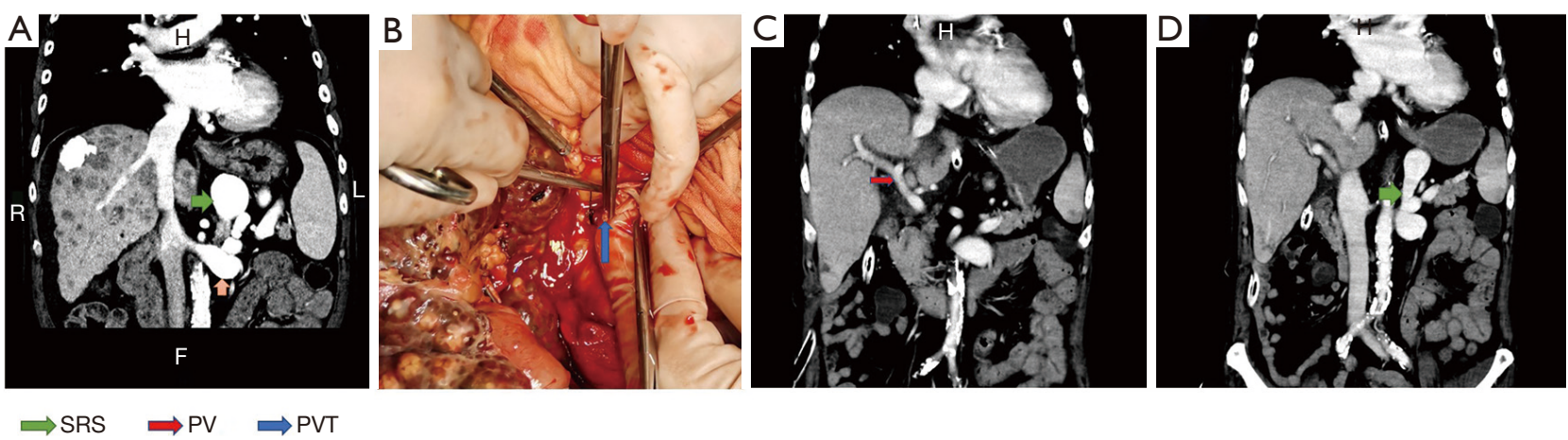

Figure 5 The perioperative image of case 2. (A) SRS with $2 \mathrm{~cm}$ in diameter between SV and LRV; (B) old PVT was removed during the operation; (C) postoperative CT showed PV and its branches were patent; (D) postoperative CT showed the diameter of SRS did not decrease. SRS, splenorenal shunt; SV, splenic vein; LRV, left renal vein; PV, portal vein; PVT, portal vein thrombus.

patients (15). Therefore, SPSS should be considered if we find HE with elevated blood ammonia while other hepatic function indexes are normal.

SPSS may have distinct effects in different liver transplant recipients. For non-small size graft, SPSS might have a portal flow "stealing" effect, which could decrease the portal flow into graft, lead to post-operative PVT and liver graft dysfunction (2). The effect of SPSS on liver graft function recovery depends on its size. SPSS could be classified as large and small size according to its maximum diameter, with a cut-off of $8 \mathrm{~mm}$ (16). SPSS with a large diameter should be ligated, and small shunts are expected to disappear after physiological splanchnic circulation restored by implantation of a normal liver. Left renal vein ligation (LRVL) is considered as a safe and effective method to increase portal flow. If the portal flow is still poor $(<1 \mathrm{~L} / \mathrm{min}$ ) after clamping the left renal vein (LRV), a renoportal anastomosis (RPA) or other surgical methods should 
be considered (17). It's worth noting that even some obvious shunts are ligated, post-operative recurrence of portal stealing phenomenon may also occur.

For small size liver graft transplantation, SPSS might have a protective effect by limiting the portal hypertransfusion and portal hypertension, which are primary causes of SFSS (4). Allard et al. found that SPSS do not negatively affect the outcome after LDLT and suggested "do-nothing" approach in living donor LT. Furthermore, dissection of the spleen or ligation of the shunts could induce additional adverse events (18). Although Gomez Gavara et al. found the incidences of post-LT HE and PVT were lower in the ligated SPSS group than those in nonligated SPSS group, they still supported the "do-nothing" idea in case of small size graft (split-liver living donor graft or small whole liver graft with low graft-to-body weight ratio) (19). Only when portal flow is deemed insufficient either on the basis of visual examination or doppler ultrasonography (portal flow velocity $<20 \mathrm{~cm} / \mathrm{s}$ or portal flow $<1 \mathrm{~L} /$ minute), the SPSS should be sought, isolated, and ligated after a positive clamping test (18). In case of spontaneous SRS, we could clamp the LRV on the left border of the IVC to test the effect of SRS on portal flow, in other case, the SPSS can be controlled directly. If the portal flow is better after SPSS clamping, the SPSS should be ligated after revascularization of the graft. Percutaneous endovascular intervention also could be considered to deal with SPSS before or after operation when needed (20).

There is no clear consensus on the "best approach" to manage SPSS in the peri-transplant period. The approach to manage SPSS should be made specifically to each institution during LT. We recommend that SPSS greater than $8 \mathrm{~mm}$ in diameter should be ligated in LT with non-small size graft to ensure adequate portal flow and preserved in LT with small size liver graft to avoid portal hypertransfusion and portal hypertension except obviously insufficient portal blood flow. We should pay attention to the changes of SPSS and take appropriate measures to deal with it when necessary in postoperative follow-up LT patients.

\section{Acknowledgments}

Funding: This work was supported by grants as follows: the National Natural Science Foundation of China (81970564, 81471583 and 81570587), the Key Clinical Specialty Construction Project of National Health and Family Planning Commission of the People's Republic of China, the Guangdong Provincial Key Laboratory Construction Projection on Organ Donation and Transplant Immunology (2013A061401007, 2017B030314018), Guangdong Provincial international Cooperation Base of Science and Technology (Organ Transplantation) (2015B050501002), Guangdong Provincial Natural Science Funds for Major Basic Science Culture Project (2015A030308010), Guangdong Provincial Natural Science Funds for Distinguished Young Scholars (2015A030306025), Special support program for training high level talents in Guangdong Province (2015TQ01R168), Pearl River Nova Program of Guangzhou (201506010014), Science and Technology Program of Guangzhou (201704020150), Sun Yat-sen University Young Teacher Key Cultivate Project (17ykzd29) and "Elite program" specially supported by China organ transplantation development foundation.

\section{Footnote}

Reporting Checklist: The authors have completed the CARE reporting checklist. Available at http://dx.doi.org/10.21037/ apm-20-2431

Peer Review File: Available at http://dx.doi.org/10.21037/ apm-20-2431

Conflicts of Interest: All authors have completed the ICMJE uniform disclosure form (available at http://dx.doi. org/10.21037/apm-20-2431). The authors have no conflicts of interest to declare.

Ethical Statement: The authors are accountable for all aspects of the work in ensuring that questions related to the accuracy or integrity of any part of the work are appropriately investigated and resolved. All procedures performed in studies involving human participants were in accordance with the ethical standards of the institutional and/or national research committee(s) and with the Helsinki Declaration (as revised in 2013). Written informed consent was obtained from the patient.

Open Access Statement: This is an Open Access article distributed in accordance with the Creative Commons Attribution-NonCommercial-NoDerivs 4.0 International License (CC BY-NC-ND 4.0), which permits the noncommercial replication and distribution of the article with the strict proviso that no changes or edits are made and the original work is properly cited (including links to both the 
formal publication through the relevant DOI and the license). See: https://creativecommons.org/licenses/by-nc-nd/4.0/.

\section{References}

1. Primignani M, Tosetti G, La Mura V. Therapeutic and clinical aspects of portal vein thrombosis in patients with cirrhosis. World J Hepatol 2015;7:2906-12.

2. Ikegami T, Shirabe K, Nakagawara H, et al. Obstructing spontaneous major shunt vessels is mandatory to keep adequate portal inflow in living-donor liver transplantation. Transplantation 2013;95:1270-7.

3. Kim SH, Lee JM, Choi JY, et al. Changes of portosystemic collaterals and splenic volume on CT after liver transplantation and factors influencing those changes. AJR Am J Roentgenol 2008;191:W8-16.

4. Man K, Fan ST, Lo CM, et al. Graft injury in relation to graft size in right lobe live donor liver transplantation: a study of hepatic sinusoidal injury in correlation with portal hemodynamics and intragraft gene expression. Ann Surg 2003;237:256-64.

5. Blei AT. Portal hypertension and its complications. Curr Opin Gastroenterol 2007;23:275-82.

6. Fernandez M, Mejias M, Garcia-Pras E, et al. Reversal of portal hypertension and hyperdynamic splanchnic circulation by combined vascular endothelial growth factor and platelet-derived growth factor blockade in rats. Hepatology 2007;46:1208-17.

7. de Franchis R; Baveno VI Faculty. Expanding consensus in portal hypertension: Report of the Baveno VI Consensus Workshop: Stratifying risk and individualizing care for portal hypertension. J Hepatol 2015;63:743-52.

8. Saad WE. Vascular anatomy and the morphologic and hemodynamic classifications of gastric varices and spontaneous portosystemic shunts relevant to the BRTO procedure. Tech Vasc Interv Radiol 2013;16:60-100.

9. Ohnishi K, Sato S, Saito M, et al. Clinical and portal hemodynamic features in cirrhotic patients having a large spontaneous splenorenal and/or gastrorenal shunt. Am J Gastroenterol 1986;81:450-5.

10. Saad WE, Lippert A, Saad NE, et al. Ectopic varices:

Cite this article as: Wang T, Gong J, Chen Z, Huang C, Yu J, Guo Z, He X. The role of spontaneous portal-systemic shunts in liver transplantation: case report and literature review. Ann Palliat Med 2021;10(7):8365-8370. doi: 10.21037/apm-20-2431 anatomical classification, hemodynamic classification, and hemodynamic-based management. Tech Vasc Interv Radiol 2013;16:158-75.

11. Saad WE. Portosystemic shunt syndrome and endovascular management of hepatic encephalopathy. Semin Intervent Radiol 2014;31:262-5.

12. Riggio O, Efrati C, Catalano C, et al. High prevalence of spontaneous portal-systemic shunts in persistent hepatic encephalopathy: a case-control study. Hepatology 2005;42:1158-65.

13. Vilstrup H, Amodio P, Bajaj J, et al. Hepatic encephalopathy in chronic liver disease: 2014 Practice Guideline by the American Association for the Study of Liver Diseases and the European Association for the Study of the Liver. Hepatology 2014;60:715-35.

14. Tomás Pujante P, Jiménez Sánchez AF, Iglesias Jorquera E, et al. Hepatic encephalopathy secondary to a splenorenal shunt that manifested a long time after a liver transplantation. Rev Esp Enferm Dig 2018;110:400-1.

15. Rogal SS, Hu A, Bandi R, et al. Novel therapy for noncirrhotic hyperammonemia due to a spontaneous splenorenal shunt. World J Gastroenterol 2014;20:8288-91.

16. Simón-Talero M, Roccarina D, Martínez J, et al. Association between portosystemic shunts and increased complications and mortality in patients with cirrhosis. Gastroenterology 2018;154:1694-705.e4.

17. Tang R, Han D, Li M, et al. Left renal vein ligation for large splenorenal shunt during liver transplantation. ANZ J Surg 2017;87:767-72.

18. Allard MA, Akamatsu N, Kokudo T, et al. Clinical significance of spontaneous portosystemic shunts in living donor liver transplantation. Liver Transpl 2021;27:77-87.

19. Gomez Gavara C, Bhangui P, Salloum C, et al. Ligation versus no ligation of spontaneous portosystemic shunts during liver transplantation: audit of a prospective series of 66 consecutive patients. Liver Transpl 2018;24:505-15.

20. Shigeta T, Kasahara M, Sakamoto S, et al. Balloonoccluded retrograde transvenous obliteration for a portosystemic shunt after pediatric living-donor liver transplantation. J Pediatr Surg 2011;46:e19-22. 